STRUCTURAL BIOLOGY COMMUNICATIONS

ISSN 2053-230X

\section{The crystal structure of the endoglucanase Cel10, a family 8 glycosyl hydrolase from Klebsiella pneumoniae}

\author{
Ayman Attigani, ${ }^{\text {a Lifang Sun, }}{ }^{\mathrm{b}}$ Qing Wang, ${ }^{\mathrm{b}}$ Yadan Liu, ${ }^{\mathrm{b}}$ Dingping Bai, ${ }^{\mathrm{a}}$ \\ Shengping $\mathrm{Li}^{\mathrm{b} *}$ and Xiaohong Huang ${ }^{\mathrm{a} *}$
}

Received 27 September 2016

Accepted 8 November 2016

Edited by W. N. Hunter, University of Dundee, Scotland

Keywords: cellulases; Klebsiella pneumoniae; carboxymethyl cellulase; cellulose biosynthesis; crystal structure; Cel10.

PDB reference: Cel10, 5gy3

Supporting information: this article has supporting information at journals.iucr.org/f

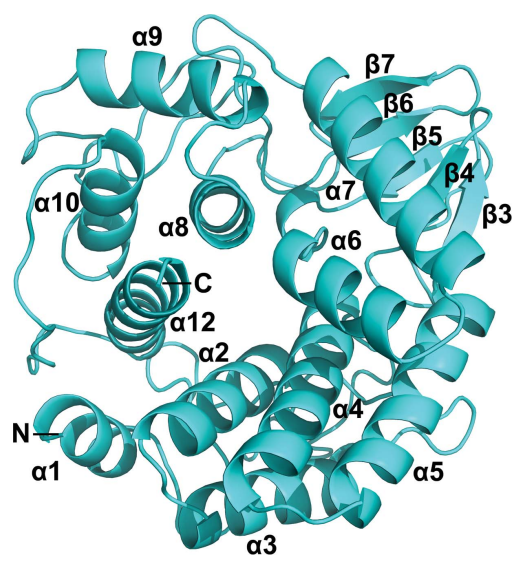

OPEN $\odot$ ACCESS
${ }^{a}$ Key Laboratory for Integrated Chinese Traditional and Western Veterinary Medicine and Animal Healthcare, College of Animal Science, Fujian Agriculture and Forestry University, 15 Shang Xia Dian Road, Fuzhou 350002, People's Republic of China, and 'b State Key Laboratory of Structural Chemistry, Fujian Institute of Research on the Structure of Matter,

Chinese Academy of Sciences, 155 West Yangqiao Road, Fuzhou 350002, People's Republic of China. *Correspondence e-mail: spingli1981@fjirsm.ac.cn, xhhuang138@hotmail.com

Cellulases are produced by microorganisms that grow on cellulose biomass. Here, a cellulase, Cel10, was identified in a strain of Klebsiella pneumoniae isolated from Chinese bamboo rat gut. Analysis of substrate specificity showed that Cel10 is able to hydrolyze amorphous carboxymethyl cellulose (CMC) and crystalline forms of cellulose (Avicel and xylan) but is unable to hydrolyze $p$-nitrophenol $\beta$-D-glucopyranoside ( $p$-NPG), proving that Cel10 is an endoglucanase. A phylogenetic tree analysis indicates that Cel10 belongs to the glycoside hydrolase 8 (GH8) subfamily. In order to further understanding of its substrate specificity, the structure of Cel10 was solved by molecular replacement and refined to $1.76 \AA$ resolution. The overall fold is distinct from those of most other enzymes belonging to the GH8 subfamily. Although it forms the typical $(\alpha / \alpha)_{6}$-barrel motif fold, like Acetobacterxylinum CMCax, one helix is missing. Structural comparisons with Clostridium thermocellum CelA (CtCelA), the best characterized GH8 endoglucanase, revealed that sugar-recognition subsite -3 is completely missing in Cel10. The absence of this subsite correlates to a more open substrate-binding cleft on the cellooligosaccharide reducing-end side.

\section{Introduction}

Cellulose is the most abundant organic compound on earth and is the major polysaccharide component of plant cell walls. Cellulose fibres comprise crystalline and amorphous arrays of polysaccharide chains. Effective hydrolysis of cellulose requires three types of cellulases, namely endo-(1,4)- $\beta$-Dglucanase (EC 3.2.1.4; carboxymethylcellulase or CMCase), exo-(1,4)- $\beta$-D-glucanase (EC 3.2.1.91; cellobiohydrolase, avicelase, microcrystalline cellulase or $\beta$-exoglucanase) and $\beta$-glucosidase (EC 3.2.1.21), which must act synergistically to achieve the degradation of crystalline cellulose (Tomme et al., 1995). Although a large number of microorganisms are capable of degrading cellulose, only a few of them produce significant quantities of cell-free bioactive compounds capable of completely hydrolyzing crystalline cellulose in vitro (Bai et al., 2012). Numerous studies have reported the degradation of cellulosic materials, but only a few have examined which microorganisms might offer economical benefits (Yamada $e t$ al., 2011). Microbes play a vital role in the degradation of cellulose and some animals have achieved effective cellulose utilization by developing symbiotic relationships with microbes that are present in their gut as the primary cellulolytic agent (Watanabe \& Tokuda, 2001). 
Table 1

Macromolecule-production information.

Source organism
DNA source
Forward primer
Reverse primer
Cloning vector
Expression vector
Expression host
Complete amino-acid sequence
of the construct product

\begin{abstract}
K. pneumoniae
cDNA from $K$. pneumoniae

CGGGATCCGATACGGCCTGGGAGCGCTA

CCGCTCGAGCTAACGCTGATCCTGTTTCG

pET-32a

pET-32a

E. coli BL21 (DE3)

GTSSMADIGSDTAWERYKARFMMPDGRIIDTANGNVSHTEGQGFAMLLAVANNDRPAFDKLWQWTDSTLRDKSNGLFYWRYNPVAPDPIADKNNASDGDTLIAWALLRAQKQWQDKRYAIASDAITASLLKYTVVTFAGRQVMLPGVKGFNLNDHLNLNPSYFIFPAWRAFAERTHLTAWRTLQTDGQALLGQMGWGKSHLPSDWVALRADGKMLPAKEWPPRMSFDAIRIPLYLSWADPQSALLAPWKAWMQSYPRLQTPAWINVSTNEVAPWYMAGGLLAVRDLTLGEPQEAPQIDDKDDYYSASLKQLVWLAKQDQR
\end{abstract}

The Chinese bamboo rat (Rhizomyssinensis) is well known for its dietary oddities: it is a bamboo specialist within the mammalian order Herbivores that possesses a gastrointestinal tract typical of carnivores. It consumes the roots and shoots of bamboo and other highly fibrous plants each day (Musser \& Carleton, 2005; Anderson \& Jones, 1984; Clarke, 2010). By sequence analysis of the conserved $16 \mathrm{~S}$ rRNA, a molecular marker for the identification of bacterial species (Srinivasan et al., 2015), a bacterium isolated from the gastrointestinal tract of the Chinese bamboo rat was identified as a Klebsiella strain and named Klebsiella 10. A cellulase gene was cloned from the Klebsiella 10 chromosomal DNA using a pair of special primers and was thus named Cel10. The gene encodes a protein of 310 amino-acid residues, including a signal-peptide segment (residues 1-23), and has a mature molecular weight of $35 \mathrm{kDa}$. The protein is predicted to be a member of glycoside hydrolase family 8 (GH8) according to the CAZy database (http://www.cazy.org). Based on the facts that genes encoding cellulases are essential in bacteria and that the proteins are putative targets for enzyme development, there have been numerous studies of the three-dimensional structures of cellulases (Dominguez et al., 1995; Ducros et al., 1995; Clarke, 2010). Previous analyses have provided a basis for modelling homologous GH8 cellulases and the architecture of the activesite cleft, which presents at least five glucosyl binding subsites and explains why GH8 cellulases cleave oligosaccharide polymers that are at least five D-glucosyl subunits in length. Furthermore, the structure of CtCelA (CelA) allows comparison with $(\alpha / \alpha)_{6}$-barrel glycosidases that are not related in sequence, suggesting a possible, albeit distant, evolutionary relationship between different families of glycosyl hydrolases (Alzari et al., 1996).

Cellulases, by virtue of their ability to degrade cellulose substantially, are key industrial enzymes of the 21st century. There is a considerable drive to uncover new enzymes, to determine their three-dimensional structures and assess them for cellulose deconstruction. Thus, recombinant Cel10 was studied in order to understand its structure-function relationship with respect to cellulolytic activity. Here, we present the cloning, expression, purification, crystallization and X-ray diffraction analysis of a cellulase from the cellulolytic bacterium $K$. pneumoniae found in the gut of the Chinese bamboo rat.

\section{Materials and methods}

\subsection{Cloning and expression of Cel10}

The DNA encoding amino acids 24-333 of Cel10 was amplified by polymerase chain reaction (PCR) using $K$. pneumoniae genomic DNA as a template and the gene-specific forward primer CLE-BamH1 (5'-CGGGATCCGATACGGCCTGGGAGCGCTA-3') and reverse primer CLE-XhoI (5'-CCGCTCGAGCTAACGCTGATCCTGTTTCG-3') (Table 1). The PCR product was cloned into the expression vector pET-32a [modified by inserting a Tobacco etch virus (TEV) protease cleavage site inside the NcoI site] with BamHI and XhoI. Escherichia coli strain DH5 $\alpha$ (Novagen) was used for plasmid amplification, which was confirmed by DNA sequencing. The recombinant plasmid was then transformed into E. coli strain BL21 (DE3) (Novagen) for protein expression. Cells were grown in Luria-Bertani (LB) medium plus $100 \mathrm{mg} \mathrm{l}^{-1}$ ampicillin with shaking at $310 \mathrm{~K}$ for $6 \mathrm{~h}$, and expression of Cel10 was induced by adding isopropyl $\beta$-D-1-thiogalactopyranoside to a final concentration of $0.3 \mathrm{~m} M$ when the cells reached the mid-log phase of growth (optical density at $600 \mathrm{~nm}$ of $0.6-0.8$ ); the cells were then grown overnight with shaking at $289 \mathrm{~K}$.

\subsection{Protein production and purification}

Cel10 protein was purified using a four-step protocol: an $\mathrm{Ni}^{2+}$-affinity chromatography step, cleavage of the N-terminal 6 His-Trx tag with TEV protease, removal of the cleaved tag by a second $\mathrm{Ni}^{2+}$-affinity chromatography step and finally sizeexclusion chromatography (SEC), which was performed on an ÄKTApurifier (GE Healthcare) using SEC programmes according to previously described procedures (Bryan et al., 2011). The cells containing expressed Cel10 were harvested by centrifugation at $7000 \mathrm{~g}$ for $5 \mathrm{~min}$ at $277 \mathrm{~K}$. The cell pellets were thawed on ice, resuspended in lysis buffer consisting of $50 \mathrm{~m} M$ MES pH 6.0, $500 \mathrm{~m} M \mathrm{NaCl}, 5 \%$ glycerol supplemented with $5 \%$ Tween 20 and $0.1 \mu \mathrm{m}$ PMSF, and disrupted by ultrasonication on ice for $30 \mathrm{~min}$. Cell debris was removed by centrifugation at $20000 \mathrm{~g}$ for $30 \mathrm{~min}$ at $277 \mathrm{~K}$ using a Beckman Avanti J-301 centrifuge. The resulting supernatant was loaded onto nickel Sepharose affinity resin. After the flowthrough had been discarded, the column was washed with lysis buffer containing a linear gradient from 20 to $100 \mathrm{~m} M$ imidazole, and target proteins were eluted from the column using lysis buffer plus $500 \mathrm{~m} M$ imidazole. 6 His-TEV protease was added to the eluted protein at a ratio of $1: 10(w: w)$ to cleave the 6His-Trx tag. The 6 His-TEV protease and 6 His-Trx tag were then removed by a second $\mathrm{Ni}^{2+}$-affinity chromatography step. The resulting protein was further purified by SEC (Superdex 200, GE Healthcare) using a buffer consisting of $50 \mathrm{~m} M$ MES, $100 \mathrm{~m} M \mathrm{NaCl} \mathrm{pH}$ 6.0, 5\% glycerol. The SEC chromatogram showed one peak at $87.69 \mathrm{ml}$ consistent with the molecular weight of Cel10 (35 kDa). After SDS-PAGE analysis (Fig. 1), 
Table 2

Crystallization.

\begin{tabular}{ll}
\hline Method & Sitting drop \\
Plate type & Cryschem plate \\
Temperature $(\mathrm{K})$ & 293 \\
Protein concentration $\left(\mathrm{mg} \mathrm{ml}^{-1}\right)$ & 28 \\
Buffer composition of protein & $20 \mathrm{~m} M$ Tris- $\mathrm{HCl} \mathrm{pH} 6.0,150 \mathrm{mM} \mathrm{NaCl}$, \\
$\quad$ solution & $5 \%$ glycerol \\
Composition of reservoir solution & $0.1 \mathrm{M}$ glycine- $\mathrm{NaOH}$ pH 9.0,30\% PEG \\
& $8 \mathrm{~K}, 0.5 \mathrm{M} \mathrm{KCl}$ \\
Volume and ratio of drop & $2 \mu \mathrm{l}(1: 1$ ratio $)$ \\
Volume of reservoir $(\mu \mathrm{l})$ & 400 \\
\hline
\end{tabular}

the purified Cel10 was concentrated for crystallization to $28 \mathrm{mg} \mathrm{ml}^{-1}$ using an ultrafiltration system (Millipore, $30 \mathrm{kDa}$ cutoff). The protein concentration was determined by the Bradford method using bovine serum albumin (BSA) as the standard (Bradford, 1976).

\subsection{Substrate specificity}

The Cel10 activity was determined according to a previously described method (Saratale et al., 2010, 2012). Endoglucanase activity was determined using a reaction mixture consisting of $1 \mathrm{ml}$ enzyme solution $\left(4 \mathrm{mg} \mathrm{ml}^{-1}\right)$ with $2 \mathrm{ml} 1 \%(w / v) \mathrm{CMC}$ in McIlvaine's buffer ( $0.1 M$ citric acid/0.2 $M$ phosphate buffer $\mathrm{pH} 5$ ) and incubated at $323 \mathrm{~K}$ for $30 \mathrm{~min}$ followed by the addition of $1.5 \mathrm{ml}$ dinitrosalicylic acid reagent. Cellulolytic activities towards Avicel for avicelase activity and towards xylan for xylanase activity were measured by replacing the

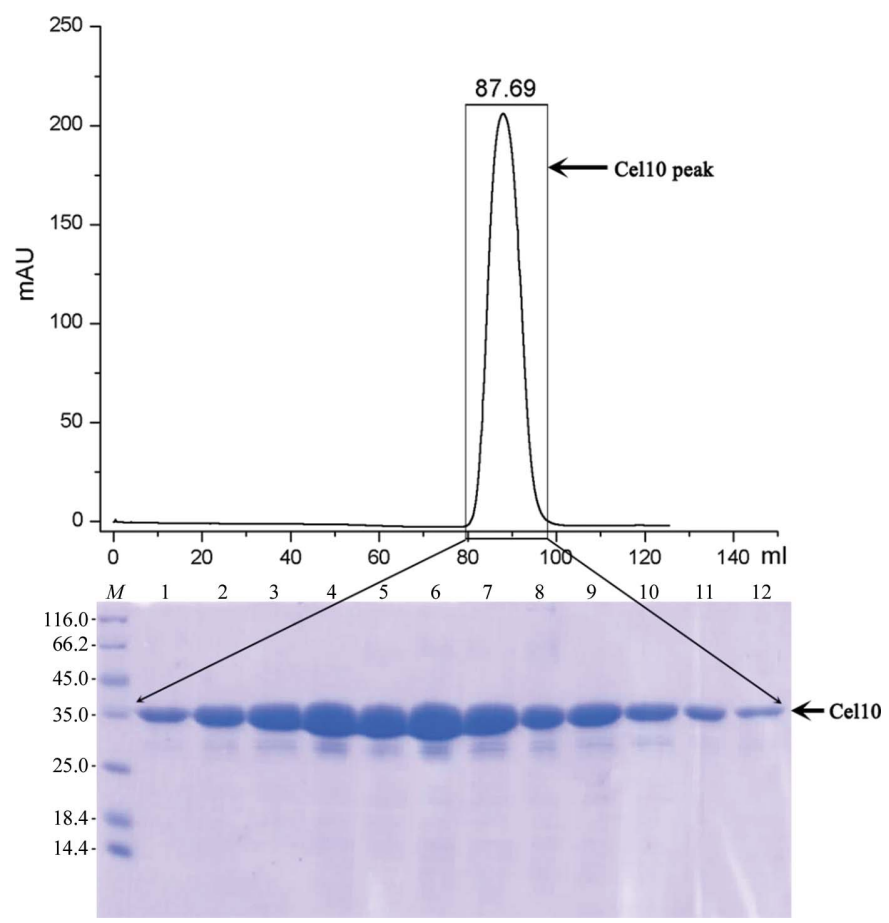

Figure 1

SDS-PAGE analysis of purified recombinant Cel10. Top: size-exclusion chromatography (SEC) chromatogram of Cel10 from the final purification column showing a notable peak. Bottom: SDS-PAGE gel of the peak fraction. The protein fractions were resolved on a gradient SDS-PAGE gel $(15 \%)$ and stained using Coomassie Blue for visualization. Lane $M$ contains molecular-weight markers (labelled in $\mathrm{kDa}$ ).
CMC from the earlier assay with $1 \%(w / v)$ of the respective substrate in the same buffer. Activities were expressed as micromole of reducing sugar (glucose or xylose) equivalent released per minute. $\beta$-Glucosidase activity was determined by measuring the hydrolysis of $p$-nitrophenyl $\beta$-D-glucopyranoside ( $p$-NPG) as described previously (Lymar et al., 1995). The enzyme $(1 \mathrm{ml})$ was incubated with $5 \mathrm{~m} M p$-NPG in $1 \mathrm{ml}$ $50 \mathrm{~m} M$ citrate buffer $\mathrm{pH} 4.5$ at $323 \mathrm{~K}$ for $60 \mathrm{~min}$, the reaction was stopped by adding $1 \mathrm{ml} 1 M$ sodium carbonate and the colour formed was measured at $410 \mathrm{~nm}$. One unit of $\beta$-glucosidase activity was defined as the amount of enzyme that liberates $1 \mu \mathrm{mol} p$-nitrophenol per minute under the assay conditions. Specific activity is defined as the number of units per milligram of protein.

\subsection{Crystallization}

Initial crystallization screening was performed at $293 \mathrm{~K}$ by the sitting-drop vapour-diffusion method using commercial crystallization screening kits. Each crystallization drop was prepared by mixing $0.3 \mu \mathrm{l}$ reservoir solution and $0.3 \mu \mathrm{l}$ protein solution, and the mixture was equilibrated against $0.1 \mathrm{ml}$ reservoir solution. After four weeks, crystals appeared in a solution consisting of PEG $8 \mathrm{~K}, 0.5 \mathrm{M}$ potassium chloride, $0.1 M$ HEPES $\mathrm{pH}$ 7.5. Conditions were further optimized by varying the $\mathrm{pH}$ value and precipitant concentrations to obtain diffraction-quality crystals (Table 2). For data collection, the crystals were grown for four weeks at $293 \mathrm{~K}$, with the optimal condition consisting of $0.1 M$ glycine- $\mathrm{NaOH}$ pH 9.0, 30\% PEG 8K, $0.5 M$ potassium chloride (Fig. 2).

\subsection{Data collection, structure determination and refinement}

Prior to data collection, a single crystal was transferred into mother liquor containing $30 \%(v / v)$ glycerol as a cryoprotectant and then mounted in a $0.1 \mathrm{~mm}$ nylon loop (Hampton Research) and flash-cooled in liquid nitrogen. X-ray diffraction data were collected to $1.76 \AA$ resolution on beamline BL17U1 at Shanghai Synchrotron Radiation Facility (SSRF;

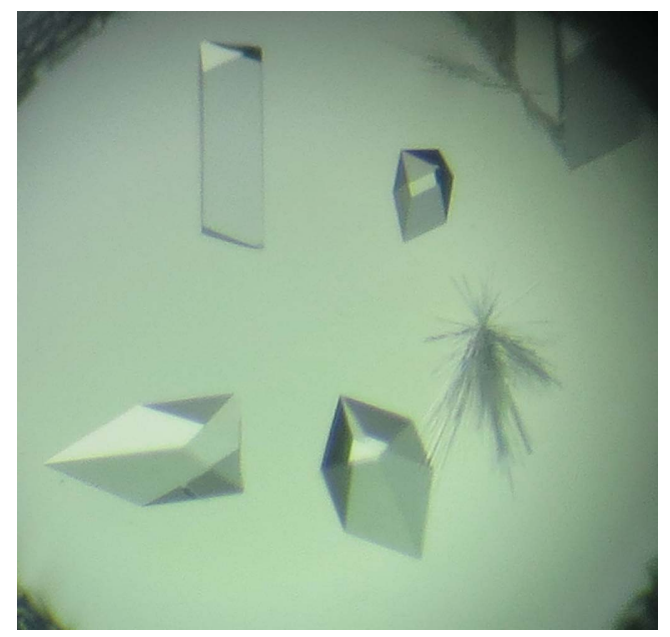

Figure 2

Crystals were obtained in $0.1 M$ glycine-NaOH pH 9.0, 30\% PEG 8K, $0.5 M$ potassium chloride by the sitting-drop vapour-diffusion method. 
Table 3

Data collection and processing.

Values in parentheses are for the outer shell.

\begin{tabular}{|c|c|}
\hline Diffraction source & BL17U1, SSRF \\
\hline Wavelength $(\AA)$ & 0.9792 \\
\hline Temperature (K) & 100 \\
\hline Detector & ADSC Q315R \\
\hline Crystal-to-detector distance $(\mathrm{mm})$ & 250 \\
\hline Rotation range per image $\left(^{\circ}\right)$ & 1 \\
\hline Total rotation range $\left({ }^{\circ}\right)$ & 180 \\
\hline Exposure time per image (s) & 0.8 \\
\hline Space group & $P 2_{1} 2_{1} 2_{1}$ \\
\hline$a, b, c(\AA)$ & $53.570,73.256,79.200$ \\
\hline$\alpha, \beta, \gamma\left({ }^{\circ}\right)$ & $90,90,90$ \\
\hline Mosaicity $\left({ }^{\circ}\right)$ & 0.3 \\
\hline Resolution range $(\AA)$ & $50-1.7639$ \\
\hline Total No. of reflections & 130639 \\
\hline No. of unique reflections & 30441 (1508) \\
\hline Completeness (\%) & 99.23 \\
\hline Multiplicity & $4.3(4.4)$ \\
\hline Wilson $B$ factor $\left(\AA^{2}\right)$ & 14.48 \\
\hline$\langle I / \sigma(I)\rangle$ & $32.98(10.02)$ \\
\hline $\mathrm{CC}_{1 / 2}$ & 0.954 \\
\hline$R_{\text {merge }} \dagger$ & $0.08(0.236)$ \\
\hline$R_{\text {r.i.m. }} \neq$ & $0.043(0.108)$ \\
\hline
\end{tabular}

$\dagger R_{\text {merge }}=\sum_{h k l} \sum_{i}\left|I_{i}(h k l)-\langle I(h k l)\rangle\right| / \sum_{h k l} \sum_{i} I_{i}(h k l)$, where $I_{i}(h k l)$ are the intensities of the individual replicates of a given reflection $h k l$ and $\langle I(h k l)\rangle$ is the average intensity over all replicates of that reflection. $\ddagger$ Estimated $R_{\text {r.im }}=R_{\text {merge }}[N /(N-1)]^{1 / 2}$, where $N$ is the data multiplicity.

Table 4

Structure determination and refinement.

\begin{tabular}{ll}
\hline Resolution range $(\AA)$ & $44.361-1.763$ \\
Completeness $(\%)$ & 99.55 \\
No. of reflections, working set & 31307 \\
No. of reflections, test set & 1517 \\
Final $R_{\text {work }}$ & 0.1615 \\
Final $R_{\text {free }}$ & 0.1988 \\
No. of non-H atoms & \\
$\quad$ Total & 2495 \\
$\quad$ Water & 480 \\
$\quad$ Total & 2975 \\
R.m.s. deviations & \\
$\quad$ Bonds $(\AA)$ & 0.006 \\
$\quad$ Angles $\left({ }^{\circ}\right)$ & 0.840 \\
Average $B$ factor $\left(\AA^{2}\right)$ & 17.0 \\
Ramachandran plot & \\
$\quad$ Favoured regions $(\%)$ & 97.08 \\
$\quad$ Additionally allowed $(\%)$ & 2.92 \\
$\quad$ Outliers $(\%)$ & 0 \\
PDB code & $5 \mathrm{gy} 3$ \\
\hline
\end{tabular}

Shanghai, People's Republic of China) using a charge-coupled device (CCD) detector. The data were processed and scaled using the $H K L-2000$ and CCP4 suites (Winn et al., 2011). Data-collection and processing statistics are shown in Table 3. The crystal belonged to space group $P 2_{1} 2_{1} 2_{1}$, with unit-cell parameters $a=53.57, b=73.26, c=79.20 \AA$, and contained one molecule in the asymmetric unit. Calculation of the Matthews coefficient using $C C P 4$ indicated a $V_{\mathrm{M}}$ of $2.22 \AA^{3} \mathrm{Da}^{-1}$, corresponding to a solvent content of $44.61 \%$. The crystal structure of Cel10 was determined by molecular replacement using the CMCax structure (PDB entry 1wzz, 36\% identity; Yasutake et al., 2006) as the search model in Phaser (McCoy et al., 2007) and was refined with PHENIX (Adams et al., 2010). All molecular figures were prepared using $P y M O L$
Table 5

Activity of endoglucanase Cel10 towards various substrates.

\begin{tabular}{lc}
\hline Substrate & Activity $\left(\mathrm{U} \mathrm{mg}^{-1}\right)$ \\
\hline CMC & 31.8 \\
Avicel & 18.3 \\
Xylan & 8.7 \\
$p$-NPG & None \\
\hline
\end{tabular}

(Schrödinger). The atomic coordinates and structure factors have been deposited in the Protein Data Bank with accession code 5gy3. Structure-refinement statistics are shown in Table 4.

\section{Results and discussion}

\subsection{Substrate-specificity analysis}

The Cel10 enzyme was analyzed using various substrates to determine its catalytic specificity, as shown in Table 5. The results showed that Cel10 hydrolyzes amorphous CMC and crystalline forms of cellulose (Avicel and xylan) but does not hydrolyze $p$-NPG. However, Cel10 cellulase activity was more efficient on CMC than on Avicel and xylan, which indicates that it is an endoglucanese. Furthermore, Cel10 was

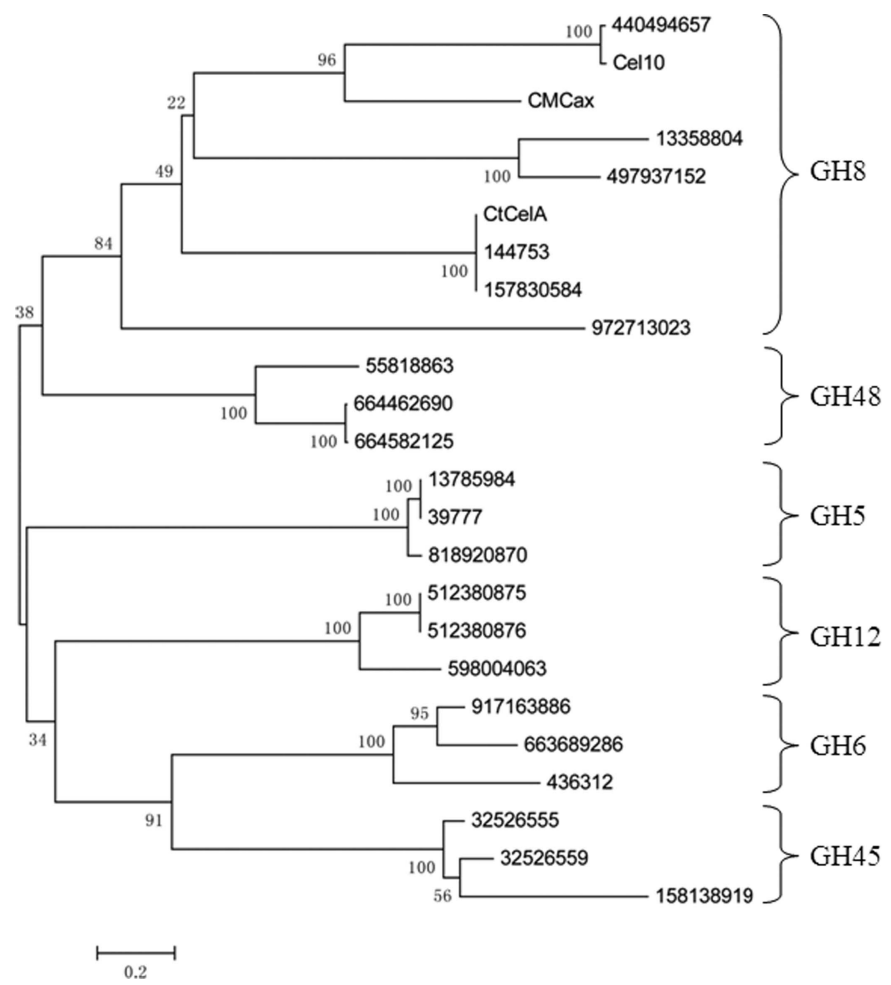

Figure 3

Phylogenetic tree showing the relationship between Cel10 and other hydrolase families. In the phylogenetic tree analysis, Cel10 was in the same cluster as members of different families and showed over $97 \%$ homology to Cel8A in GH8 (GenBank accession No. 440494657). These results demonstrate that Cel10 is a member of GH8. The phylogenetic tree was drawn using $M E G A$ v.4.0. The amino-acid sequence of Cel10 was aligned with those from other different cellulase hydrolase families to generate a neighbour-joining phylogenetic tree. Bootstrap percentage values are indicated at branch points. Accession numbers are listed in the centre. 
considered to be a member of the GH8 family according to the CAZy database (http://www.cazy.org; Cantarel et al., 2009) and in a phylogenetic analysis with MEGA4.0 (Tamura et al., 2007) from amino-acid sequence comparison of Cel10 with other glycosyl hydrolase family members (Fig. 3). CMC (an amorphous cellulose derivative) is commonly used as a substrate for the study of endoglucanases (Lynd et al., 2002). On the other hand, exoglucanases can degrade Avicel efficiently (Lynd et al., 2002). In our case, Cel10 displayed a stronger catalytic preference for CMC than for Avicel. In a comparison of activity against CMC with other endoglucanases (Schwarz et al., 1986; Mahadevan et al., 2008), CtCelA showed the highest enzyme specific activity. Interestingly, the differences between these recombinant endoglucanases illustrate that the enzymatic activity was mainly affected by the original strain specificity (Posta et al., 2004), the classification of the GH family (Janeček et al., 2011) and synergism (Lynd et al., 2002).

Our data suggest that the degree of hydrolysis of an insoluble substrate might be related to intermolecular synergy between the carbohydrate-binding module (CBM) and the catalytic domain of cellulases, which occurs because binding of the CBM to the cellulose substrate brings the catalytic domain to the substrate surface and the CBM loosens the crystalline structure by partially separating the cellulose strands from the surface of cellulose microfibrils, making the substrate easier to hydrolyze (Lynd et al., 2002). Therefore, CBM is essential for the hydrolysis of crystalline cellulose (Ogawa et al., 2007). It has been proposed that these independent 'domains' are critical for targeting the enzymes to the substrate and for enhancing their hydrolytic activity. This result suggests that the absence of a CBD in Cel10 makes it less effective against crystalline cellulose.

\subsection{Three-dimensional structure of Cel10}

The structure of Cel10 was solved by molecular replacement using the three-dimensional structure of the Acetobacterxylinum endoglucanase CMCax (PDB entry 1wzz) as the search model. The final structure was refined at $1.76 \AA$ resolution with an $R_{\text {work }}$ of $16.15 \%$ and an $R_{\text {free }}$ of $19.88 \%$ (Table 4). There is one molecule in the asymmetric unit and the final structure contains residues 24-333. The structure of Cel10 is mainly composed of 11 helices forming an overall socalled 'barrel fold' ( $\alpha 1-\alpha 12$; Fig. $4 a$ ), which differs from most of the other enzymes belonging to the GH8 subfamily, which display an atypical $(\alpha / \alpha)_{6}$-barrel motif fold. It is similar to the CMCax structure but with one helix ( $\alpha 11$ in CtCelA, labelled in red) missing in the Ce110 structure; instead a flexible loop is formed (labelled green) (Fig. 4b). Notably, compared with the flexible loops in the CtCelA structure the connections between helices $\alpha 5$ and $\alpha 6$ and between $\alpha 7$ and $\alpha 8$ form extended $\beta$-strands $\beta 3, \beta 4$ and $\beta 5$, and $\beta 6$ and $\beta 7$, respectively, with two antiparallel $\beta$-sheets being formed by $\beta 3, \beta 4$ and $\beta 5$ and by $\beta 6$ and $\beta 7$ (Fig. 5). However, the functional role of this stable protrusion, which differs from that in the corresponding part of CtCelA, awaits further investigation. As reported, CtCelA is one of the best-characterized endo- $\beta-1,4$-glucanases, with the structure having been determined in complex

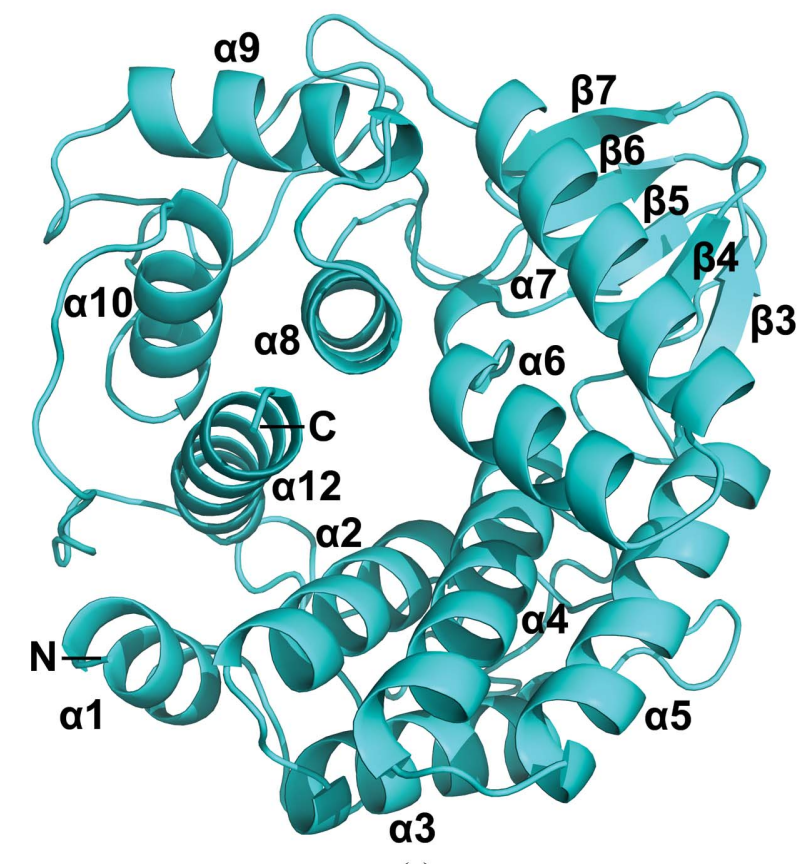

(a)

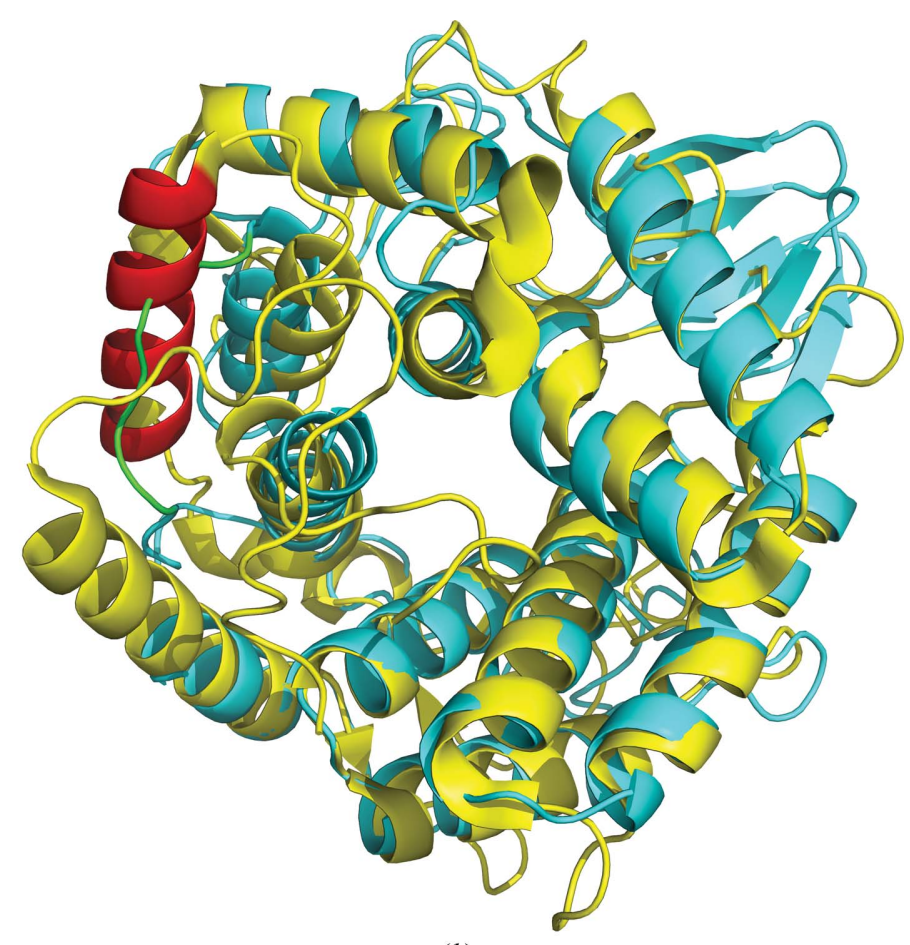

(b)

Figure 4

Structure of Cel10 ( $a$ ) and structure superimposition of Cel10 (PDP entry 5gy3; blue) with CtCelA (PDB entry 1 kwf; yellow) (b). Helix $\alpha 11$ in CtCelA (labelled in red) is missing and forms a flexible loop (labelled in green) in Ce110. 
with the cellobiose substrate (Alzari et al., 1996). According to the phylogenetic analysis, Cel10 should exhibit essentially similar enzymatic characteristics to CtCelA (Fig. 3). However, structural comparisons of the active sites of Cel10 and CtCelA reveal notable differences. Two of the five aromatic residues involved in stacking interactions that are critical for substrate

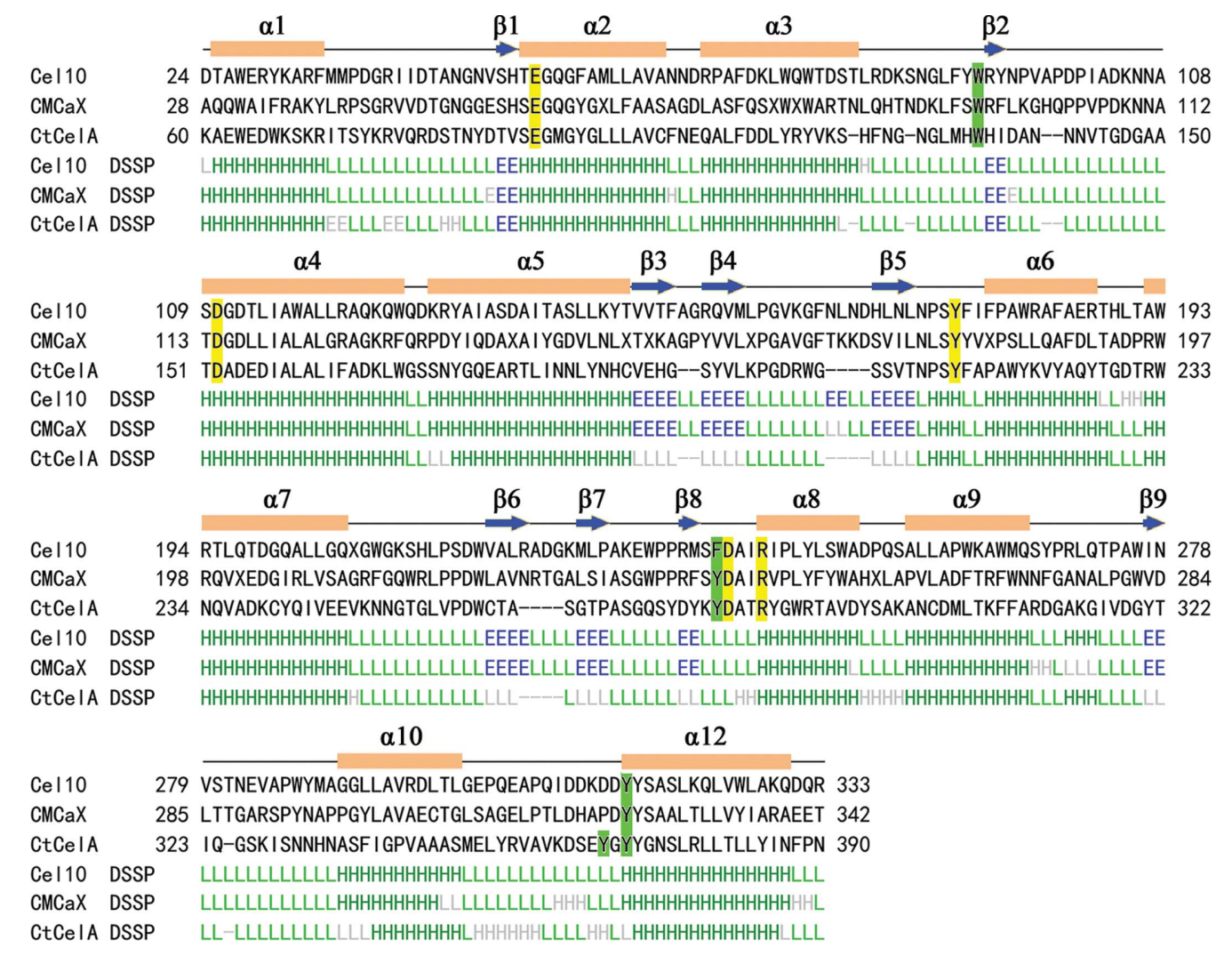

Figure 5

The structure and sequence alignment of Cel10 with CMCax (PDB entry 1wzz) and CtCelA (PDB entry 1kwf). Structure-based sequence alignment of enzymes belonging to GH8. Conserved catalytic residues are highlighted in yellow and the aromatic residues forming sugar-recognition subsites are shown in green. This figure was created using DaliLite (Holm \& Rosenström, 2010).

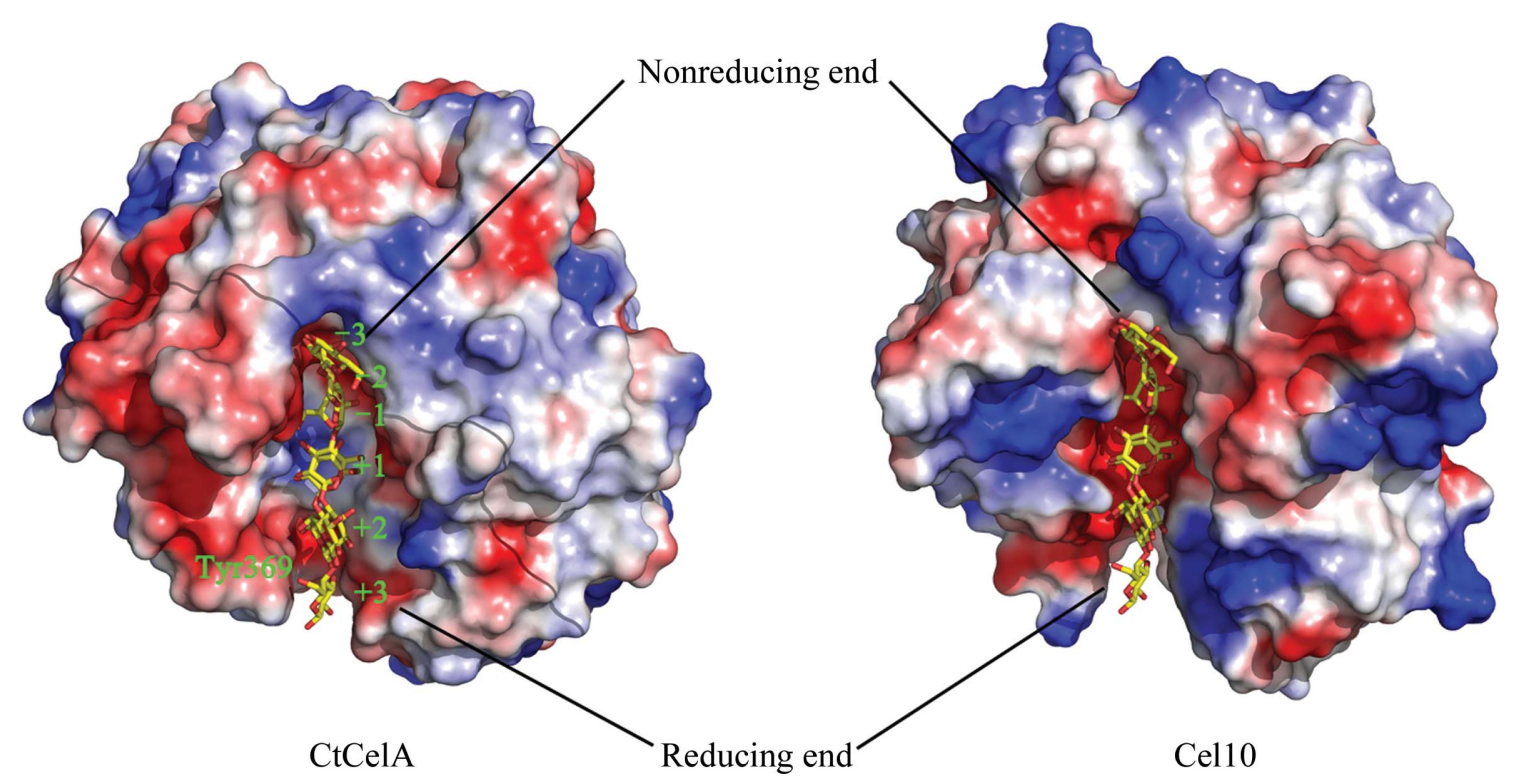

Figure 6

Molecular surface-potential representation of CtCelA (left; PDB entry 1kwf) and Cel10 (right). A model of the substrate in PDB entry $1 \mathrm{kwf}$ is also shown in the cleft of Cel10. The electrostatic surface potentials were generated using PyMOL (http://www.pymol.org) in absolute mode. Areas coloured white, red and blue denote neutral, negative and positive potential, respectively. 
recognition by CtCelA (Guérin et al., 2002), corresponding to Trp205 and Tyr369 of CtCelA, are not conserved in Cel10. Phe163 of Cel10 seems to play an identical role to Trp205 of CtCelA, while a residue corresponding to Tyr369 of CtCelA is missing in the Cel10 structure, leading to a significant broadening of the cleft at the cellooligosaccharide reducing end (Fig. 6). These observations suggested that sugar-recognition subsite -3 is not present in Cel10, implying that Cel10 cannot immobilize cellobiose at the active-site cleft owing to the structural differences in the oligosaccharide recognition site.

Consistent with the homology model of CMCax, Populus tremula $\times$ tremuloides $\mathrm{KOR}$ and the expected structure of AgCelC (Master et al., 2004), the absence of subsite -3 of Cel10 is a common feature among cellulose biosynthesisrelated endoglucanases (Yasutake et al., 2006). It has been speculated that KOR may function in cleavage of the lipidlinked glucose from the reducing end of the growing glucan chain (Peng et al., 2002), and it has been reported that AgCelC may act as a transferase rather than as an endoglucanase during cellulose synthesis (Matthysse et al., 1995). The absence of subsite -3 may account for the recognition of such lipidlinked oligosaccharides. However, the relationship between cellulose synthesis and lipid-linked oligosaccharides in A. xylinum has not yet been clarified, and the actual role of Cel10 in the cellulose-production process requires further investigation.

\section{Acknowledgements}

The authors thank the staff of beamline BL17U1 at Shanghai Synchrotron Radiation Facility (SSRF) for support during diffraction data collection. This work was supported by the Natural Science Foundation of Fujian Province (2013J01151), a Key Project of Fujian Province (2014H0056), the Fujian Provincial Department of Science and Technology (No. KH2130004) and the National Nature Science Foundation of China (31470741 and 31270790).

\section{References}

Adams, P. D. et al. (2010). Acta Cryst. D66, 213-221.

Alzari, P. M., Souchon, H. \& Dominguez, R. (1996). Structure, 4, 265-275.

Anderson, S. \& Jones, J. K. (1984). Orders and Families of Recent Mammals of the World. New York: John Wiley \& Sons.

Bai, S., Kumar, M. R., Kumar, D. M., Balashanmugam, P., Kumaran, M. \& Kalaichelvan, P. (2012). Arch. Appl. Sci. Res. 4, 269-279.

Bradford, M. M. (1976). Anal. Biochem. 72, 248-254.

Bryan, C. M., Bhandari, J., Napuli, A. J., Leibly, D. J., Choi, R., Kelley, A., Van Voorhis, W. C., Edwards, T. E. \& Stewart, L. J. (2011). Acta Cryst. F67, 1010-1014.
Cantarel, B. L., Coutinho, P. M., Rancurel, C., Bernard, T., Lombard, V. \& Henrissat, B. (2009). Nucleic Acids Res. 37, D233-D238.

Clarke, N. D. (2010). Curr. Opin. Struct. Biol. 20, 527-532.

Dominguez, R., Souchon, H., Spinelli, S., Dauter, Z., Wilson, K. S., Chauvaux, S., Béguin, P. \& Alzari, P. M. (1995). Nature Struct. Biol. 2, 569-576.

Ducros, V., Czjzek, M., Belaich, A., Gaudin, C., Fierobe, H.-P., Belaich, J.-P., Davies, G. J. \& Haser, R. (1995). Structure, 3, 939-949.

Guérin, D. M., Lascombe, M. B., Costabel, M., Souchon, H., Lamzin, V., Béguin, P. \& Alzari, P. M. (2002). J. Mol. Biol. 316, 1061-1069.

Holm, L. \& Rosenström, P. (2010). Nucleic Acids Res. 38, W545W549.

Janeček, Š., Svensson, B. \& MacGregor, E. A. (2011). Enzyme Microb. Technol. 49, 429-440.

Lymar, E. S., Li, B. \& Renganathan, V. (1995). Appl. Environ. Microbiol. 61, 2976-2980.

Lynd, L. R., Weimer, P. J., van Zyl, W. H. \& Pretorius, I. S. (2002). Microbiol. Mol. Biol. Rev. 66, 506-577.

Mahadevan, S. A., Wi, S. G., Lee, D.-S. \& Bae, H. J. (2008). FEMS Microbiol. Lett. 287, 205-211.

Master, E. R., Rudsander, U. J., Zhou, W., Henriksson, H., Divne, C., Denman, S., Wilson, D. B. \& Teeri, T. T. (2004). Biochemistry, 43, 10080-10089.

Matthysse, A. G., Thomas, D. L. \& White, A. R. (1995). J. Bacteriol. 177, 1076-1081.

McCoy, A. J., Grosse-Kunstleve, R. W., Adams, P. D., Winn, M. D., Storoni, L. C. \& Read, R. J. (2007). J. Appl. Cryst. 40, 658-674.

Musser, G. \& Carleton, M. (2005). Mammal Species of the World: A Taxonomic and Geographic Reference, edited by D. E. Wilson \& D. M. Reeder, pp. 894-1531. Baltimore: Johns Hopkins University Press.

Ogawa, A., Suzumatsu, A., Takizawa, S., Kubota, H., Sawada, K., Hakamada, Y., Kawai, S., Kobayashi, T. \& Ito, S. (2007). J. Biotechnol. 129, 406-414.

Peng, L., Kawagoe, Y., Hogan, P. \& Delmer, D. (2002). Science, 295, 147-150.

Posta, K., Béki, E., Wilson, D. B., Kukolya, J. \& Hornok, L. (2004). J. Basic Microbiol. 44, 383-399.

Saratale, G. D., Saratale, R. G., Lo, Y.-C. \& Chang, J.-S. (2010). Biotechnol. Prog. 26, 406-416.

Saratale, G. D., Saratale, R. G. \& Oh, S. E. (2012). Biomass Bioenergy, 47, 302-315.

Schwarz, W. H., Gräbnitz, F. \& Staudenbauer, W. L. (1986). Appl. Environ. Microbiol. 51, 1293-1299.

Srinivasan, R., Karaoz, U., Volegova, M., MacKichan, J., KatoMaeda, M., Miller, S., Nadarajan, R., Brodie, E. L. \& Lynch, S. V. (2015). PLoS One, 10, e0117617.

Tamura, K., Dudley, J., Nei, M. \& Kumar, S. (2007). Mol. Biol. Evol. 24, 1596-1599.

Tomme, P., Warren, R. A. \& Gilkes, N. R. (1995). Adv. Microb. Physiol. 37, 1-81.

Watanabe, H. \& Tokuda, G. (2001). Cell. Mol. Life Sci. 58, 1167-1178.

Winn, M. D. et al. (2011). Acta Cryst. D67, 235-242.

Yamada, R., Taniguchi, N., Tanaka, T., Ogino, C., Fukuda, H. \& Kondo, A. (2011). Biotechnol. Biofuels, 4, 8.

Yasutake, Y., Kawano, S., Tajima, K., Yao, M., Satoh, Y., Munekata, M. \& Tanaka, I. (2006). Proteins, 64, 1069-1077. 